\title{
Markov Regime Switching of Stochastic Volatility Lévy Model on Approximation Mode
}

\author{
Arthit Intarasit ${ }^{1,2}$ \\ ${ }^{1}$ Department of Mathematics and Computer Science, Faculty of Science and Technology, Prince of Songkla University, Pattani Campus, \\ 181 Charoenpradit Road, Rusamelae, Muang, Pattani 94000, Thailand \\ ${ }^{2}$ Centre of Excellence in Mathematics, CHE, Si Ayutthaya Road, Bangkok 10400, Thailand
}

Correspondence should be addressed to Arthit Intarasit; iarthit@bunga.pn.psu.ac.th

Received 11 August 2013; Accepted 27 October 2013

Academic Editor: Ferenc Hartung

Copyright ( $\odot 2013$ Arthit Intarasit. This is an open access article distributed under the Creative Commons Attribution License, which permits unrestricted use, distribution, and reproduction in any medium, provided the original work is properly cited.

This paper deals with financial modeling to describe the behavior of asset returns, through consideration of economic cycles together with the stylized empirical features of asset returns such as fat tails. We propose that asset returns are modeled by a stochastic volatility Lévy process incorporating a regime switching model. Based on the risk-neutral approach, there exists a large set of candidates of martingale measures due to the driving of a stochastic volatility Lévy process in the proposed model which renders the market incomplete in general. We first establish an equivalent martingale measure for the proposed model introduced in risk-neutral version. Regime switching of stochastic volatility Lévy process is employed in an approximation mode for model calibration and the calibration of parameters model done based on EM algorithm. Finally, some empirical results are illustrated via applications to the Bangkok Stock Exchange of Thailand index.

\section{Introduction}

The market of stock products is one of the fastest growing main segments in the finance market industry today. Although the financial credit derivative industry has increased in market size, particular stock market sector investments are still attractive for all investors. We can see that in the Stock Exchange of Thailand, between 2011 to 2013, the Bangkok Stock Exchange of Thailand (SET) index, a major stock market index tracking the performance of all common stocks listed on the SET market, showed high volatility in movement behavior. The SET index average went up about $36 \%$ : from 1,025 index points in 2011 to 1,392 index points in 2012, with fluctuations in its movement in 2013. Historically, the SET averaged 727 index points from 1987 to 2013, with an all time record high of 1754 index points in January of 1994 and a record low of 207 index points in September of 1998. Figure 1 displays historical value of SET index with the sample period from January 2011 to February 2013.

A pricing of SET index movement that takes into account fluctuation and high volatility has become necessary. We need models that can capture the behavior of asset prices more accurately in order to handle trade risks. Recently, continuous-time financial models have been intensively investigated in explorations to capture the stylized empirical features of asset prices or returns such as long memory, fat tails, high kurtosis, volatility clustering, and leverage. On the other hand, a new generation of financial models are able to reproduce the different phase of the business cycles and capture the cyclical behavior of the economic growth. Known as regime switching models, they were first proposed by Hamilton [1]. Succeeding Hamilton's regime switching models, quite a few number of researchers have utilized the regime switching approach to help their models become more realistic in parameter estimation and forecasting accuracy. Thus the class of regime switching models has extended in many directions.

In their development of a continuous framework, Elliott et al. [2] proposed the use of the exponential of a pure jump process with finite states of a Markov-switching compensator. On the other hand, Siu et al. [3] generalized a jump diffusion model with a Markov-switching compensator and used it to value participating life insurance products. Combining stochastic volatility models together with regime switching 


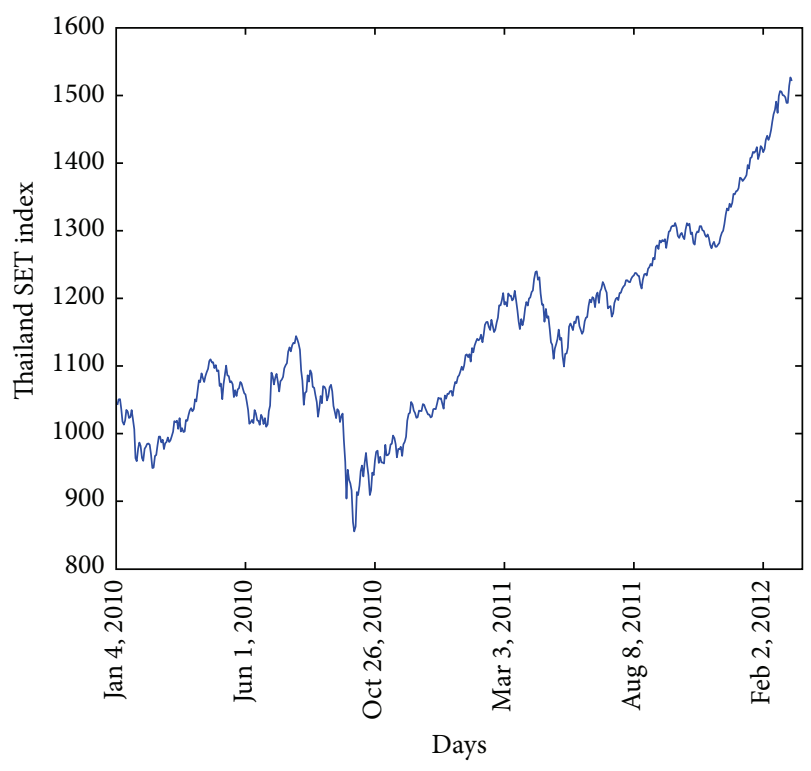

(a)

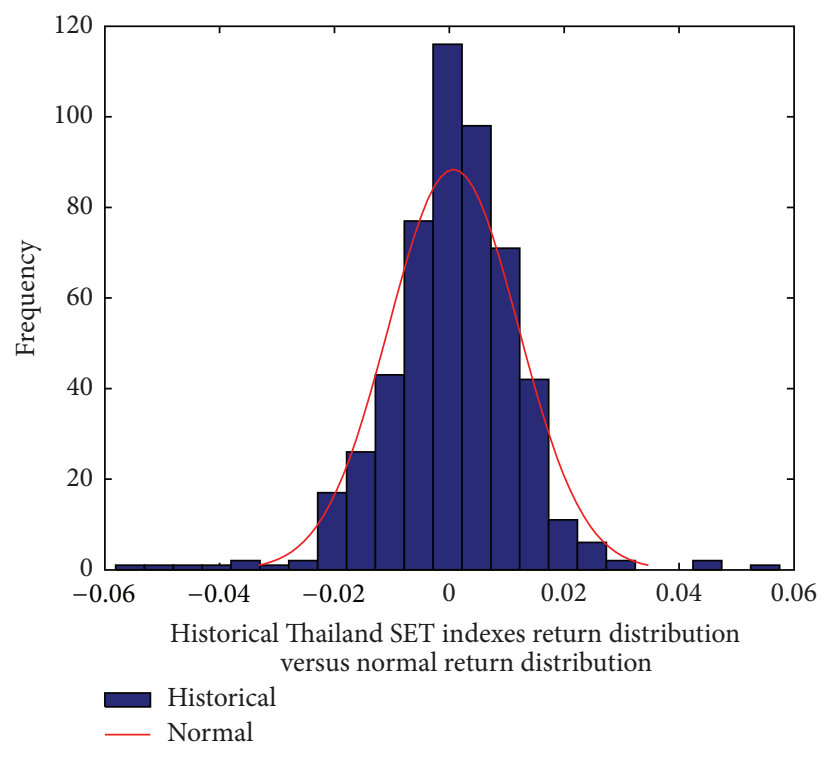

(b)

FIGURE 1: Historical value of SET index with sample period from January 2011 to February 2013 (a) and empirical distribution of daily log returns for the SET index and fitted normal distribution (b).

has been modeled for short-term interest rates, for example, Kalimipalli and Susmel [4] and Smith [5]. Janczura and Weron [6] discussed the calibration models based on the $\mathrm{EM}$ algorithm built on the mean-reverting process combined with Markov regime switching.

It is know that Lévy processes are a class of stochastic processes that help us capture a financial asset aspect of a more realistic model such as the phenomenon of jump in asset prices or the implied volatility smile in option markets, showing that the risk-neutral returns are nonGaussian and leptokurtic. Although this recent modeling of asset returns by jump diffusion allows for regime switching, few studies have explored models of diffusion with Lévy jump incorporating stochastic volatility and switching in regimes for modeling an asset return. Motivated by this fact, we propose a jump diffusion process including its variance as a stochastic volatility and the asset return considered on Markov's regime switching models to facilitate the matching of the empirical distribution with the asset return founded in real economic data. Under the risk-neutral approach, we construct and study our proposed model in a risk-neutral world. Approximation of proposed model with special structures is presented to avoid complexity of numerical computation and to suggest a suitable consistent approximation model of the proposed model.

The rest of paper is organized as follows. In Section 2, we introduce the dynamic of asset return described by the stochastic volatility Lévy process. In fact, the model is a bivariate-stochastic differential equation, whose solution of proposed dynamics is obtained by using the Itô formula for Lévy processes. Using the approach of martingale modeling, we construct a martingale measure and introduce the riskneutral model of proposed model in Section 3. In Section 4 model specification for implementation is introduced and its approximation is considered in Section 5. We state our proposed model incorporating regime switching in Section 6. Finally, we present some empirical results from applying our model to real market financial data.

\section{Modeling Asset Price Dynamics}

In order to model financial asset prices in the market, we introduce Lévy jump diffusion (LJD) with stochastic volatility (SV), a bivariate-stochastic differential equation (SDE) type, as follows.

Let $W=\left(W_{1}, W_{2}\right)$ be a two-dimensional standard Brownian motion on a filtered probability space $(\Omega, \mathscr{F}, P, F)$ with filtration $F=\left(\mathscr{F}_{t}\right)_{0 \leq t \leq T}$, the $P$-augmentation of the filtration generated by $W$. We consider two financial assets $(B, S)$ with a risk-free asset (such as a bank account or bond) with price dynamics described by

$$
d B_{t}=r_{t} B_{t} d t, \quad B_{0}=1,
$$

where the interest-rate process $r: \Re \rightarrow \Re$ is continuous. The return process of the risky asset $S$ is described by a diffusion model to incorporate the Lévy jump; its variance is an $\mathrm{SV}$ process as in the following general form:

$$
\begin{gathered}
d S_{t}=\left(\mu_{t}-q_{t}\right) S_{t-} d t+\sigma\left(v_{t}\right) S_{t-} d W_{1, t}+S_{t-} d Z_{t}, \\
d v_{t}=a\left(t, S_{t}, v_{t}\right) d t+b\left(t, S_{t}, v_{t}\right) d W_{2, t},
\end{gathered}
$$

where the stock-drift process $\mu:[0, T] \rightarrow \mathfrak{R}$ and the dividend-yield process $q:[0, T] \rightarrow \Re$ are continuous processes, and $\sigma(\cdot)$ is an SV process. The notation $S_{t-}$ is defined by

$$
S_{t-}:=\lim _{s \uparrow t} S_{s}
$$


which means that whenever there is a jump, the value of the process before the jump is used on the left-hand side of (2). The last term of (2), the differential form of the jump derived by the Lévy process, is defined in the form of Lévy-Itô decomposition as

$$
Z_{t}:=\int_{0}^{t} \int_{\mathfrak{R}} z \bar{N}(d s, d z)
$$

where

$$
\bar{N}(d t, d z)= \begin{cases}N(d t, d z)-v(d z) d t=: \widetilde{N} & \text { if }|z|<1 \\ N(d t, d z) & \text { if }|z| \geq 1\end{cases}
$$

Let $\mathbf{B}_{0}$ be the family of Borel sets $A \subset \Re$ whose closure $\bar{A}$ does not contain 0 . Let $\chi_{A}$ denote the indicator function of the set $A$. Let $A \in \mathbf{B}_{0}$ and the process $N$ be a Poisson random measure of $S(\cdot)$ in $\mathfrak{R}_{+} \times \mathfrak{R}_{0}\left(\mathfrak{R}_{0}:=\mathfrak{R} \backslash\{0\}\right)$ defined by

$$
N((s, t] \times A):=\sum_{u \in(s, t]: \Delta S \neq 0} \chi_{A}\left(\Delta S_{u}\right)
$$

with Lévy measure $v$ of the price process $S$ given by

$$
\nu(A):=E[N(1, A)]=E\left[\sum_{u \in(s, t]: \Delta S \neq 0} \chi_{A}\left(\Delta S_{u}(\omega)\right)\right] .
$$

Here $E=E_{P}$ denotes the expectation with respect to measure $P$. The jump process $\Delta S:=\left(\Delta S_{t}\right)_{0 \leq t \leq T}$ associated with the price process $S$ is defined, for each $0 \leq t \leq T$, via $\Delta S_{t}:=$ $S_{t}-S_{t-}$ where $S_{t-}$ is defined as (4). The process $\widetilde{N}$ is called the compensated Poisson random measure.

Given a correlation process $\rho$ between $d W_{1, t}$ and $d W_{2, t}$ we introduce the Brownian motion $d \widetilde{W}_{2, t}$ independent of $d W_{1, t}$ and write the usual Cholesky factorization:

$$
d W_{2, t}=\rho d W_{1, t}+\sqrt{1-\rho^{2}} d \widetilde{W}_{2, t}
$$

with correlation $\rho \in(-1,1)$.

Furthermore we assume that all processes are bounded and sufficiently smooth to guarantee unique strong solutions of the various stochastic differential equations that we encounter.

The following proposition provides an explicit solution of $\operatorname{SDE}(2)$.

Proposition 1. Suppose that some risky assets have a dynamics of return given by $S D E$ (2) with an initial value $S_{0}=S(0)$ almost everywhere (a.s.). Under the historical measure $P$, the asset price $S_{t}$ at time $t$ is then given by

$$
\begin{aligned}
S_{t}=S(0) \exp \{ & \int_{0}^{t}\left(\mu_{s}-q_{s}-\frac{1}{2} \sigma^{2}\left(v_{s}\right)\right) d s+\int_{0}^{t} \sigma\left(v_{s}\right) d W_{1, s} \\
& +\int_{0}^{t} \int_{|z|<1}\{\ln (1+z)-z\} \nu(d z) d s \\
& \left.+\int_{0}^{t} \int_{\mathfrak{R}} \ln (1+z) \bar{N}(d s, d z)\right\} .
\end{aligned}
$$

Proof. If we define the process $X_{t}=\ln S_{t}$, such that $S_{t}$ corresponds to SDE (2), then it follows from the Itô's formula (see [7, Theorem 1.14]) with $S_{0}=S(0)$ a.s. that the process (10) is the explicit solution of $\operatorname{SDE}(2)$.

The process $S_{t}$ of (10) is called the stochastic volatility of geometric Lévy model (SVGL) which is used to model the process of risky asset pricing in this paper.

\section{Risk-Neutral Dynamics of Asset Price}

Incorporating a Lévy jump and/or stochastic volatility in a diffusion model of asset returns leads to a market being incomplete. As a result there are different choices of equivalent martingale measure. By risk-neutral modeling, we should determine the dynamic price of asset return in the risk-neutral version and choose a pricing measure form various equivalent martingale measures.

We write as usual $\widetilde{S}_{t}=S_{t} / B_{t}$ for the discounted stock price process with the bank account being the natural numéraire and get from Itô's formula again

$$
d \widetilde{S}_{t}=\left(\mu_{t}-q_{t}-r_{t}\right) \widetilde{S}_{t-} d t+\sigma\left(v_{t}\right) \widetilde{S}_{t-} d W_{1, t}+\widetilde{S}_{t-} d \widetilde{Z}_{t},
$$

where the Lévy jump martingale $\widetilde{Z}_{t}$ is defined by

$$
\widetilde{Z}_{t}=\int_{0}^{t} \int_{\Re} z \widetilde{N}(d s, d z),
$$

and the process $v_{t}$ is given by $\operatorname{SDE}(3)$. The process $\widetilde{N}$ is the compensated Poisson random measure as defined in (6).

To determine the equivalent martingale measure $Q$ under which discounted price processes $\widetilde{S}_{t}$ are (local) $\mathscr{F}_{t^{-}}$ martingales, we rely on Girsanov's theorem for semimartingales (see, e.g., [8, Theorem 7.4.1]). In our model, this follows from the special structure of the Girsanov density used to perform a change of measure in setting of both Brownian motion and Lévy jump. Define the market price process of risk $\theta_{1, t}:=\mu_{t}-q_{t}-r_{t}$. For $\varepsilon>0$ define the approximation process of the process $\theta_{1, t}$ by

$$
\theta_{\varepsilon, t}:=\frac{\mu_{\varepsilon, t}-q_{\varepsilon, t}-r_{\varepsilon, t}}{\sigma\left(v_{t}\right)},
$$

where $\mu_{\varepsilon}, q_{\varepsilon}$, and $r_{\varepsilon}$ are continuous functions from $[0, T]$ to $\mathfrak{R}$, which are the approximation functions of $\mu, q$, and $r$, respectively. Assume in addition that the total mass of the Lévy jump measure $v$ exceeds $\theta_{1, t}-\theta_{\varepsilon, t}$ so that

$$
\int_{\mathfrak{R}} \nu(d z)>\frac{\theta_{1, t}-\sigma\left(v_{t}\right) \theta_{\varepsilon, t}}{z} .
$$

By this assumption, we define

$$
\theta_{3, z}:=\frac{\left(\theta_{1, t}-\sigma\left(v_{t}\right) \theta_{\varepsilon, t}\right)}{z \nu(A)} \chi_{A}(z),
$$

where $A \subset \Re$ with $\theta_{1, t}-\sigma\left(v_{t}\right) \theta_{\varepsilon, t}<\nu(A)<\infty$. For $\varepsilon>0$, fix $\theta_{\varepsilon, t}, \theta_{1, t}$, and $\theta_{3, z}<1$ such that a martingale condition satisfies

$$
\theta_{1, t}-\theta_{\varepsilon, t} \sigma\left(v_{t}\right)-\int_{\Re} z \theta_{3, z} \nu(d z)=0,
$$


setting

$$
\theta_{2, t}=-\frac{1}{\sqrt{1-\rho^{2}}}\left(\rho \theta_{\varepsilon, t}+\frac{\theta_{1, t}}{b\left(t, S_{t}, v_{t}\right)}\right),
$$

so that the process $L_{t}$ defined by

$$
\begin{aligned}
\log L_{t}= & -\int_{0}^{t} \theta_{1, s} d W_{1, s}-\int_{0}^{t} \theta_{2, s} d W_{2, s}-\frac{1}{2} \int_{0}^{t}\left(\theta_{1, s}^{2}+\theta_{2, s}^{2}\right) d s \\
& +\int_{0}^{t} \int_{\Re} \ln \left(1-\theta_{3, z}\right) \widetilde{N}(d s, d z) \\
& +\int_{0}^{t} \int_{\Re}\left[\ln \left(1-\theta_{3, z}\right)+\theta_{3, z}\right] \nu(d z) d s
\end{aligned}
$$

exists for $0 \leq t \leq T$. Define a probability measure $Q$ on $\mathscr{F}_{T}$ by

$$
d Q(\omega)=L(T) d P(\omega)
$$

Assume that

$$
E_{P}[L(T)]=1
$$

Then $Q$ is an equivalent local martingale measure for $\widetilde{S}_{t}$.

Under $Q$, let us assume that the Novikov condition holds; that is,

$$
E_{P}\left[\exp \left(\frac{1}{2} \int_{0}^{T} \theta_{i, s}^{2} d s\right)\right]<\infty,
$$

where $i=1,2$. The notation $E_{P}$ then denotes the expectation with respect to the measure $P$. Then the processes

$$
\begin{aligned}
& W_{1, t}^{\mathrm{Q}}=W_{1, t}+\int_{0}^{t} \theta_{\varepsilon, s} d s, \\
& \widetilde{W}_{2, t}^{\mathrm{Q}}=\widetilde{W}_{2, t}+\int_{0}^{t} \theta_{2, s} d s
\end{aligned}
$$

are Brownian motions with respect to $Q$ so that we define the random measure $\widetilde{N}_{Q}(\cdot, \cdot)$ by

$$
\widetilde{N}_{Q}(d t, d z):=N(d t, d z)-v_{Q}(d t, d z),
$$

where the Lévy measure $v_{\mathrm{Q}}$ is given by

$$
v_{\mathrm{Q}}(d t, d z)=\left(1-\theta_{3, z}\right) \nu(d z) d t
$$

with a $P$-intensity measure $\nu(d z) d t$ and function $\theta_{3, z}$ defined as above and satisfying the martingale condition (16). Then

$$
\begin{aligned}
\int_{0}^{t} \int_{\mathfrak{R}} \widetilde{N}_{\mathrm{Q}}(d s, d z)= & \int_{0}^{t} \int_{\mathfrak{R}} N(d s, d z) \\
& -\int_{0}^{t} \int_{\mathfrak{R}}\left(1-\theta_{3, z}\right) \nu(d z) d s
\end{aligned}
$$

is a $Q$ local martingale.
Under the risk-neutral measure $Q$, we rewrite the process of discounted stock price (11) in terms of these processes:

$$
\begin{aligned}
\frac{d \widetilde{S}_{t}}{\widetilde{S}_{t-}}= & \left(\mu_{t}-q_{t}-r_{t}\right) d t+\sigma\left(v_{t}\right) d W_{1, t}+\int_{\Re} z \widetilde{N}(d t, d z) \\
= & \left(\mu_{t}-q_{t}-r_{t}-\theta_{\varepsilon, t} \sigma\left(v_{t}\right)\right) d t+\sigma\left(v_{t}\right) d W_{1, t}^{\mathrm{Q}} \\
& +\int_{\Re} z N(d s, d z)-\int_{\mathfrak{R}} z \nu(d z) d t \\
= & \left(\theta_{1, t}-\theta_{\varepsilon, t} \sigma\left(v_{t}\right)\right) d t+\sigma\left(v_{t}\right) d W_{1, t}^{\mathrm{Q}}-\int_{\mathfrak{R}} z \nu(d z) d t \\
& +\int_{\mathfrak{R}} z\left\{\widetilde{N}_{\mathrm{Q}}(d s, d z)+\left(1-\theta_{3, s}(z)\right) \nu(d z) d t\right\}
\end{aligned}
$$

so that the process of discounted price follows

$$
\begin{gathered}
d \widetilde{S}_{t}=\widetilde{S}_{t-}\left\{\left(\theta_{1, t}-\theta_{\varepsilon, t} \sigma\left(v_{t}\right)-\int_{\Re} z \theta_{3, s} \nu(d z)\right) d t\right. \\
\left.+\sigma\left(v_{t}\right) d W_{1, t}^{Q}+\int_{\Re} z \widetilde{N}_{Q}(d s, d z)\right\} .
\end{gathered}
$$

Using the martingale condition (16), the process $\widetilde{S}_{t}$ is a local martingale under $Q$ and

$$
d \widetilde{S}_{t}=\widetilde{S}_{t-}\left(\sigma\left(v_{t}\right) d W_{1, t}^{\mathrm{Q}}+\int_{\mathfrak{R}} z \widetilde{N}_{\mathrm{Q}}(d s, d z)\right),
$$

or equivalently,

$$
d S_{t}=S_{t-}\left(r_{t} d t+\sigma\left(v_{t}\right) d W_{1, t}^{\mathrm{Q}}+\int_{\mathfrak{R}} z \widetilde{N}_{\mathrm{Q}}(d s, d z)\right) .
$$

Therefore the dynamic of the $(S, v)$ under the risk-neutral martingale measure $Q$ is

$$
\begin{aligned}
d S_{t}=S_{t-} & \left(r_{t} d t+\sigma\left(v_{t}\right) d W_{1, t}^{\mathrm{Q}}+\int_{\Re} \widetilde{N}_{\mathrm{Q}}(d s, d z)\right) \\
d v_{t}= & \left(a\left(t, S_{t}, v_{t}\right)+\theta_{1, t}\right) d t \\
& \quad+b\left(t, S_{t}, v_{t}\right)\left(\rho_{t} d W_{1, t}^{\mathrm{Q}}+\sqrt{1-\rho_{t}^{2}} d \widetilde{W}_{2, t}^{\mathrm{Q}}\right) .
\end{aligned}
$$

Proposition 2. Suppose that the asset return process $S_{t}$ is governed by SED (30). Define $\widetilde{X}_{t}=\ln S_{t}$; then under the riskneutral measure $Q$,

$$
\begin{aligned}
d \widetilde{X}_{t}= & \int_{0}^{t}\left(r_{s}-\frac{1}{2} \sigma^{2}\left(v_{s}\right)\right) d t \\
& +\int_{|z|<1}\{\ln (1+z)-z\} v_{\mathrm{Q}}(d t, d z)+\int_{0}^{t} \sigma\left(v_{s}\right) d W_{1, s}^{\mathrm{Q}} \\
& +\int_{\mathfrak{R}} \ln (1+z) \widetilde{N}_{\mathrm{Q}}(d t, d z) .
\end{aligned}
$$

Proof. In a similar way to that of Proposition 1 , the proof follows from the Itô formula applied to $\widetilde{X}_{t}=\ln \left(S_{t}\right)$. We omit the details. 


\section{Model Specification for Implementation}

Both of the SDEs (2) or (30) together with (3) or (31), respectively, are expressed in an abstract form in a very general setting enabling many different processes of asset price to be used. Here, we will use a jump diffusion process with variance following a square root of a stochastic process as an example. The full model is

$$
\begin{gathered}
d S_{t}=S_{t-}\left((\mu-q) d t+\sqrt{v_{t}} d W_{1, t}+\int_{\Re} z \bar{N}(d s, d z)\right), \\
d v_{t}=\beta\left(m-v_{t}\right) d t+\sigma \sqrt{v_{t}} d W_{2, t},
\end{gathered}
$$

where $m$ is the rate of reversion computed by the long run mean of the process $v:=\left(v_{t}\right)_{t \geq 0}$ and $\beta$ is the log rate at which $v$ tends to $m$. The risk-neutral counterparts to these equations are, respectively,

$$
\begin{aligned}
& d S_{t}=S_{t-}\left(r d t+\sqrt{v_{t}} d W_{1, t}^{\mathrm{Q}}+\int_{\Re} z \widetilde{N}_{\mathrm{Q}}(d s, d z)\right), \\
& d v_{t}=\left((\mu-q-r)+\beta\left(m-v_{t}\right)\right) d t+\sigma \sqrt{v_{t}} d W_{2, t}^{\mathrm{Q}},
\end{aligned}
$$

where $d W_{2, t}^{\mathrm{Q}}$ is defined in a similar way to (9) on a martingale measure $Q$. All subscripted variables with risk-neutral measure $Q$ represent risk-neutral versions of the actual variables. By Proposition 1 and definition (6) of $\bar{N}$, the SDE (33) has solution:

$$
\begin{aligned}
S_{t}=S(0) \exp \left\{\int_{0}^{t}\left(\mu-q-\frac{1}{2} v_{s}\right) d s+\int_{0}^{t} \sqrt{v_{s}} d W_{1, s}\right. \\
+\int_{0}^{t} \int_{|z|<1}\{\ln (1+z)-z\} \nu(d z) d s \\
+\int_{0}^{t} \int_{|z|<1} \ln (1+z) \widetilde{N}(d s, d z) \\
\left.+\int_{0}^{t} \int_{|z| \geq 1} \ln (1+z) N(d s, d z)\right\} .
\end{aligned}
$$

The process $N$ is a Poisson random measure in $\mathfrak{R}_{+} \times \mathfrak{R}_{0}$ with Lévy measure $v(d t, d x)=v(d x) d t$, for a measure $v$ satisfying $\int_{\mathfrak{R}_{0}} \min \left(1, z^{2}\right) v(d z)<\infty$. The last term of (37),

$$
\int_{0}^{t} \int_{|z| \geq 1} \ln (1+z) N(d s, d z),
$$

representing the sum of the big jumps, is a compound Poisson process with intensity of jumps $\nu(|z|>1)$ and jump distribution $\chi(|z| \geq 1) \nu(d x) / \nu(|z| \geq 1)$. Over finite intervals $[0, t]$ this sum is finite since any cádlág path of Lévy process $S$ has only finite number of big jumps with absolute jump size larger than 1 . The third term of (37) is the limit of compensated Poisson processes as follows: for $\varepsilon>0$,

$$
\begin{aligned}
& \int_{0}^{t} \int_{|z|<1} \ln (1+z) \widetilde{N}(d s, d z) \\
& \quad=\lim _{\varepsilon \downarrow 0} \int_{0}^{t} \int_{\varepsilon<|z|<1} \ln (1+z) \widetilde{N}(d s, d z)
\end{aligned}
$$

represents the sum of the small jumps. In general there are too many jumps to get convergence but this sum can converge by compensating. Moreover, the sum of the small jumps of the solution of SDE (35) converges due to the jump part of SED (35) driven by the compensated risk-neutral measure $Q$.

Here we use the approximation theorem for the distribution of Lévy process developed in [9] but modify it in accordance with our model (37). Let $\varepsilon>0$ be given and define the following processes:

$$
\begin{aligned}
S_{t, \varepsilon}^{\ddagger}=S(0) \exp \left\{\int_{0}^{t} b_{s, \varepsilon} d s+\int_{0}^{t} \sqrt{v_{s}} d W_{1, s}\right. \\
\left.+\int_{0}^{t} \int_{|z| \geq \varepsilon} \ln (1+z) N(d s, d z)\right\},
\end{aligned}
$$

where $b_{s, \varepsilon}:=\mu-q-(1 / 2)\left(v_{s}\right)-\int_{\varepsilon<|z| \leq 1}\{\ln (1+z)-z\} \nu(d z)$, and

$$
\begin{aligned}
S_{t, \varepsilon}:=S_{t}-S_{t, \varepsilon}^{\ddagger}= & \int_{0}^{t} \int_{|z|<1}\{\ln (1+z)-z\} \nu(d z) d s \\
& +\int_{0}^{t} \int_{|z|<\varepsilon} \ln (1+z) \widetilde{N}(d s, d z) .
\end{aligned}
$$

Define $\sigma_{\varepsilon}^{2}:=\int_{|z| \leq \varepsilon} z^{2} \nu(d z)$ and suppose the $v$ has no atoms in a neighborhood of the origin. Introduce a candidate approximation of SDE (37) in distribution sense:

$$
\begin{aligned}
S_{t, \varepsilon}^{\dagger}=S(0) \exp \left\{\int_{0}^{t} b_{s, \varepsilon} d s+\int_{0}^{t} \sqrt{v_{s}^{2}+\sigma_{\varepsilon}^{2}} d W_{1, s}\right. \\
\left.+\int_{0}^{t} \int_{|z| \geq \varepsilon} \ln (1+z) N(d s, d z)\right\} .
\end{aligned}
$$

If

$$
\lim _{\varepsilon \rightarrow 0} \frac{1}{\varepsilon} \sigma_{\varepsilon}=\infty,
$$

then the process $\left(v_{\varepsilon}^{-1} S_{t, \varepsilon}\right)_{t \geq 0}$ converges in distribution to a standard Brownian motion $\left(B_{t}\right)_{t \geq 0}$, and by (43), it holds that

$$
\sup _{x \in \Re}\left|P\left(S_{t} \leq x\right)-P\left(S_{t, \varepsilon}^{\dagger} \leq x\right)\right| \leq c \frac{\varepsilon}{\sigma_{\varepsilon}} .
$$

Therefore, the distribution of $S_{t}$ (37) (with infinite jump activity) can be approximated closely to $S_{t, \varepsilon}^{\dagger}$, the combination of a Wiener process with drift and a compound Poisson process.

An approximation to the solution of SED (35) may be obtained in the same way.

\section{Approximation of Asset Price Dynamics}

We begin by finding an explicit formula of the stochastic process $v=\left(v_{t}\right)_{0 \leq t \leq T}$, where $v_{t}$ satisfies the SED (36) for simulation purposes. The process $v_{t}$ is obtained using stochastic calculus transformation techniques [10, Theorem 1]. Let $V_{t}=$ $F\left(t, v_{t}\right) \in C^{1,2}(\mathfrak{R})$. Applying Itô's lemma to the process $V$, we obtain the following equation:

$$
d V_{t}=F_{t}\left(t, v_{t}\right) d t+F_{v}\left(t, v_{t}\right) d v_{t}+\frac{1}{2} F_{v v}\left(t, v_{t}\right) \sigma_{v}^{2} v_{t} d t
$$


Then the solution of the stochastic process $v$ is

$$
v_{t}=e^{-\beta}\left(\frac{V_{t}}{2}\right)^{2}
$$

where

$$
\begin{gathered}
V_{t}=2 \sqrt{v_{0}}+2 I_{g}(t) \\
I_{g}(t)=\frac{1}{2} \int_{0}^{t} e^{\beta / 2}\left(\left(\frac{\beta m-\sigma^{2} / 4}{\sqrt{v_{s}}}\right) d s+\sigma d W_{2, s}^{\mathrm{Q}}\right) .
\end{gathered}
$$

Calibration and simulation are done in a discrete time framework. For simulation purposes using the forward Euler discretization scheme, we divide the time horizon $[0, T]$ of $T$ years into $N_{0} T$ subintervals of equal length $\Delta:=T / N_{0}$, where $T$ is a positive integer. For each $n=1, \ldots, N_{0}$, the $n$th subinterval is represented by $\left[t_{n}, t_{n-1}\right]$ where $t_{n}=n \Delta$. Let $v_{n}$ denote $v_{t_{n}}$. The following proposition shows that the simulation of the volatility process $v$ is best performed by using (46).

Proposition 3. The exact value of the realization of the solution $v$ at time $t_{n+1}$ is generated by the following reclusive formula:

$$
v_{n+1}=v_{0}+2 \sqrt{v_{n}} \widetilde{I}_{g}\left(t_{n}\right)+\widetilde{I}_{g}^{2}\left(t_{n}\right)
$$

where

$$
\begin{gathered}
\widetilde{I}_{g}\left(t_{n}\right)=\frac{1}{2} e^{\beta / 2}\left(\frac{\left(\beta m-\sigma^{2} / 4\right) \Delta_{n}}{\sqrt{v_{n}}}+\Delta W_{2, n}^{\mathrm{Q}}\right) \\
\Delta W_{2, n}^{\mathrm{Q}}=\int_{t_{n}}^{t_{n+1}} d W_{2, t}^{\mathrm{Q}}=W_{2, t_{n+1}}^{\mathrm{Q}}-d W_{2, t_{n}}^{\mathrm{Q}}
\end{gathered}
$$

is the $\mathcal{N}\left(0, \Delta_{n}\right)$ distributed increment of the Brownian motion $W$ on $\left[t_{n}, t_{n+1}\right]$ and

$$
\Delta_{n}=\int_{t_{n}}^{t_{n+1}} d t=t_{n+1}-t_{n}
$$

is the length of the time discretization subinterval $\left[t_{n}, t_{n+1}\right]$.

For numerical experiments, we simplified implementation to generate processes $v$ that only apply explicit schemes to (48).

The discretized version of the risk-neutral log return process $S_{t, \varepsilon}^{\dagger}$ is deduced from the approximation of each component as follows: let $\Delta>0$ be given and let $X_{t}^{\dagger}:=\log S_{t, \varepsilon}^{\dagger}$. Rewrite the $S_{t, \varepsilon}^{\dagger}$ for log returns:

$$
\begin{aligned}
X_{t+\Delta}^{\dagger}= & X_{t}^{\dagger}+\int_{t}^{t+\Delta} b_{s, \varepsilon} d s+\int_{t}^{t+\Delta} \sqrt{v_{s}^{2}+v_{\varepsilon}^{2}} d W_{1, s} \\
& +\int_{t}^{t+\Delta} \int_{|z| \geq \varepsilon} \ln (1+z) N(d s, d z) .
\end{aligned}
$$

As in the Euler scheme, an integrand over $[t, t+\Delta]$, is approximated by its value at $t$. We now approximate the first integral term using

$$
\int_{t}^{t+\Delta} b_{s, \varepsilon} d s \approx b_{t, \varepsilon} \Delta
$$

Following the first integral term, we get

$$
\int_{t}^{t+\Delta} \sqrt{v_{s}^{2}+v_{\varepsilon}^{2}} d W_{1, s} \approx\left(\sqrt{v_{t}^{2}+v_{\varepsilon}^{2}}\right) \Delta W_{1, t},
$$

with $\Delta W_{1, t}:=W_{1, t+\Delta}-W_{1, t}$. From the remaining integral in (51), we get

$$
\int_{t}^{t+\Delta} \int_{|z| \geq \varepsilon} \ln (1+z) \Delta N(d s, d z) \approx\left(\Delta_{N} S_{t, \varepsilon}^{\dagger}\right) \Delta,
$$

where $\Delta_{N} S_{t, \varepsilon}^{\dagger}$ is the jump process of $S_{t, \varepsilon}^{\dagger}$ caused by the jump of $N(t, z)$, as denoted by

$$
\Delta_{N} S_{t, \varepsilon}^{\dagger}=\int_{|z| \geq \varepsilon} \ln (1+z) N(t, d z) .
$$

Note that this process consists of the (possible) jumps in $X$ at $t$ arising from the jump in $N(t, \cdot)$.

With these approximations, the discretized version of the risk-neutral $\log$ return process $X_{t}$ is displayed in the following proposition.

Proposition 4. Based on Euler approximation, the discretization scheme for $S_{t, \varepsilon}^{\dagger}$ is as follows:

$$
\begin{gathered}
S_{n+1, \varepsilon}^{\dagger}=S_{n, \varepsilon}^{\dagger} \exp \left\{b_{n, \varepsilon} \Delta_{n}+\sqrt{v_{n}^{2}+v_{\varepsilon}^{2}}\left(\sqrt{\Delta_{n}} Z_{n+1}\right)\right. \\
\left.+\left(\Delta_{N} S_{n, \varepsilon}^{\dagger}\right) \Delta_{n}\right\}
\end{gathered}
$$

with $v_{n}$ following from the scheme (48).

Proof. We use the approximation of (52), (53), and (54) to approximate $S_{t+\Delta, \varepsilon}^{\dagger}$. With the transformation $S^{\dagger}=\exp \left(X^{\dagger}\right)$, the discretized version of the risk-neutral $S_{t+\Delta, \varepsilon}^{\dagger}$ is

$$
S_{t+\Delta, \varepsilon}^{\dagger} \approx S_{t, \varepsilon}^{\dagger} \exp \left\{b_{t, \varepsilon} \Delta+\sqrt{v_{t}^{2}+v_{\varepsilon}^{2}} \Delta W_{1, t}+\left(\Delta_{N} S_{t, \varepsilon}^{\dagger}\right) \Delta\right\} .
$$

We apply this recursively at any $\Delta=\Delta_{n}:=t_{n+1}-t_{n}>0$ and replace the increment of Brownian motion $W$ with $\sqrt{\Delta} Z, Z \sim$ $\mathscr{N}(0,1)$. More explicitly, we have (56).

From (56) and setting $X_{t, \varepsilon}^{\dagger}:=\log S_{t+\Delta, \varepsilon}^{\dagger}$, we have

$$
X_{t+\Delta, \varepsilon}^{\dagger} \approx X_{t}^{\dagger}+b_{t, \varepsilon} \Delta+\sqrt{v_{t}^{2}+v_{\varepsilon}^{2}} \Delta W_{1, t}+\left(\Delta_{N} S_{t, \varepsilon}^{\dagger}\right) \Delta .
$$

We can now calibrate the model parameters in this equation. Let us consider the parameter set for $X^{\dagger}$, given by $\Theta=$ $\left(r, \mu, \beta, m, \sigma, \lambda, \sigma_{Y}\right)$. The remaining parameters $q$ are fixed that calibrate the parameter based on historical data.

The conditionals of the process $X^{\dagger}$ are normal and independent, due to the locally constancy of the SV $v$ on small $\Delta t$. The log-likelihood function for the asset return $X_{n}^{\dagger}$ and $v_{n}$ is then given by

$$
\mathscr{L}(\Theta)=\sum_{n=1}^{N_{0}} \log f\left(X_{n}^{\dagger}, v_{n} ; \Theta\right) .
$$


The probability density function (pdf) of process $X_{n}$ given $X_{n-1}$ together with the volatility $v_{n}$ given $v_{n-1}$ has a conditional Gaussian distribution

$$
\begin{aligned}
f\left(x_{n+1}^{\dagger}, v_{n+1} \mid \Theta\right)=\sum_{j=0}^{+\infty} & \mathbb{Q}\left(n_{t}=j\right) \\
\times & f_{\mathcal{N}}\left(x_{n+1}^{\dagger}, v_{n+1} ; b_{n, \varepsilon} \Delta_{n}+x_{n}^{\dagger}\right. \\
& \left.+j \mu_{Y}, \widehat{v}_{n}^{2} \Delta_{n}+j \sigma_{Y}^{2}\right),
\end{aligned}
$$

where $\widehat{v}_{n}:=\sqrt{v_{n}^{2}+v_{\varepsilon}^{2}}$ and

$$
\begin{aligned}
f_{\mathcal{N}}\left(x_{n+1}^{\dagger}, v_{n+1} ; \Theta\right) \\
\quad=\frac{1}{\sqrt{2 \pi\left(\hat{v}_{n}^{2}+j \sigma_{Y}^{2}\right)}} \exp \left(-\frac{\left(x_{n+1}^{\dagger}-b_{n, \varepsilon}-x_{n}^{\dagger}-j \mu_{Y}\right)^{2}}{2\left(\widehat{v}_{n}^{2}+j \sigma_{Y}^{2}\right)}\right) .
\end{aligned}
$$

This pdf is the sum of the conditional probability densities weighted by the probability of the conditioning variables of the number of jumps $n_{t}=N_{t}-N_{t-\Delta t}$ which counts the jumps between time $t-\Delta t$ and $t$. Observe that if $\Delta t$ is small, typically the Poisson process jumps at most one as a result of a mixture of two Gaussian random variables weighted by the probability of zero or one jump in $\Delta t$. Then

$$
\begin{aligned}
f\left(x_{n+1}^{\dagger} ; \Theta\right) & \\
= & \left(1-\lambda t_{n+1}\right) f_{\mathcal{N}}\left(x_{n+1}^{\dagger}, v_{n+1} ; b_{n, \varepsilon} \Delta_{n}+x^{\dagger}, \hat{v}_{n}^{2} \Delta_{n}\right) \\
& +\lambda t_{n+1} f_{\mathcal{N}}\left(x_{n+1}^{\dagger}, v_{n+1} ; b_{n, \varepsilon} \Delta_{n}+x_{n}^{\dagger}+\mu_{Y}, \hat{v}_{n}^{2} \Delta_{n}+\sigma_{Y}^{2}\right) .
\end{aligned}
$$

\section{The Switching-Regime Model of Asset Price Dynamics}

We described our proposed model whose parameter values depend on the value of a continuous-time Markov chain.

Let $\left(\alpha_{t}\right)_{t \in[0, T]}$ be a continuous-time Markov chain on finite space $\mathcal{S}:=\{1,2, \ldots, K\}$ and the transition rate matrix $Q=\left[q_{i j}\right]$ where the transition rate $q_{i j}$ is calculated with respect to the natural filtration generated by continuous-time Markov chain $\alpha, \mathscr{F}_{t}^{\alpha}:=\left\{\sigma\left(\alpha_{s}\right): 0 \leq s \leq t\right\}$ and given by

$$
\begin{gathered}
q_{i j} \geq 0 \quad \text { if } i \neq j \forall i, j \in \mathcal{S}, \\
q_{i i}=-\sum_{j=0, j \neq i}^{K} q_{i j} \text { otherwise. }
\end{gathered}
$$

By definition, the Markov chains $\alpha_{t}$ have the probability of a transition from state $i$ to state $j$ not depending on the global time and depending only on the time interval available for the transition; that is,

$$
\mathbb{P}\left(\alpha_{t+s}=j \mid \alpha_{s}=i\right)=q_{i j}=\mathbb{P}\left(\alpha_{t}=j \mid \alpha_{0}=i\right)
$$

for all $s, t \geq 0$. The regime switching (RS) of SVL model (35)(36) is defined by the following SDE:

$$
\begin{aligned}
d S_{t}= & S_{t-}\left(r\left(\alpha_{t}\right) d t+\sqrt{v_{t}} d W_{1, t}^{\mathrm{Q}}+\int_{\mathfrak{R}} z \widetilde{N}_{\mathrm{Q}}(d s, d z)\right), \\
d v_{t}= & \left(\mu\left(\alpha_{t}\right)-q\left(\alpha_{t}\right)-r\left(\alpha_{t}\right)+\beta\left(\alpha_{t}\right)\left(m\left(\alpha_{t}\right)-v_{t}\right)\right) d t \\
& +\sigma\left(\alpha_{t}\right) \sqrt{v_{t}} d W_{2, t}^{\mathrm{Q}} .
\end{aligned}
$$

The processes $r\left(\alpha_{t}\right), \mu\left(\alpha_{t}\right), q\left(\alpha_{t}\right), \beta\left(\alpha_{t}\right), m\left(\alpha_{t}\right)$, and $\sigma\left(\alpha_{t}\right)$ are constants taking values, respectively, in $r(\mathcal{S}), \mu(\mathcal{S}), q(\delta)$, $\beta(\mathcal{S}), m(\mathcal{S})$, and $\sigma(\mathcal{S})$ such that

$$
\begin{aligned}
r(\mathcal{S}):=\left\{r_{1}, \ldots, r_{K}\right\}, & \mu(\mathcal{S}):=\left\{\mu_{1}, \ldots, \mu_{K}\right\}, \\
q(\mathcal{S}):=\left\{q_{1}, \ldots, q_{K}\right\}, & \beta(\mathcal{S}):=\left\{\beta_{1}, \ldots, \beta_{K}\right\}, \\
m(\mathcal{S}):=\left\{m_{1}, \ldots, m_{K}\right\}, & \sigma(\mathcal{S}):=\left\{\sigma_{1}, \ldots, \sigma_{K}\right\} .
\end{aligned}
$$

The solution $S_{t}\left(\alpha_{t}\right)$ of (65) with SV (66) is called an RS of SVGL model. Let $\mathscr{F}_{n}^{S}$ be the filtration of historical value of the process $S_{n}$ untial time $t_{n}$. By Proposition 4 , its approximation of the solution $S_{t}\left(\alpha_{t}\right)$ in discrete version is given by

$$
\begin{gathered}
S_{n+1, \varepsilon}^{\dagger}=S_{n, \varepsilon}^{\dagger} \exp \left\{b_{n, \varepsilon}\left(\alpha_{n}\right) \Delta_{n}+\sqrt{v_{n}^{2}\left(\alpha_{n}\right)+v_{\varepsilon}^{2}}\left(\sqrt{\Delta_{n}} Z_{n+1}\right)\right. \\
\left.+\left(\Delta_{N} S_{n, \varepsilon}^{\dagger}\right) \Delta_{n}\right\} .
\end{gathered}
$$

The regime switching version of model (33) with (34) and its solution are defined by in similar manner.

In the case that the future regime of the economy has only 2 states $\alpha_{k}=k, k=1,2$, the conditional distribution density of $X_{n+1, \varepsilon}^{\dagger}$, the log price of $S_{n+1, \varepsilon}^{\dagger}$, has associated parameters across regimes given by

$$
\begin{aligned}
f\left(X_{n+1, \varepsilon}^{\dagger} \mid \mathscr{F}_{n}^{\alpha} ; \Theta\right)= & f\left(X_{n+1, \varepsilon}^{\dagger} \mid \mathscr{F}_{n}^{\alpha}, \alpha_{n}=1 ; \Theta_{1}\right) q_{1, n} \\
& +f\left(X_{n+1, \varepsilon}^{\dagger} \mid \mathscr{F}_{n-1}^{\alpha}, \alpha_{n}=2 ; \Theta_{2}\right)\left(1-q_{1, n}\right)
\end{aligned}
$$

up to the conditional probability $q_{1, n}=Q\left\{\alpha_{n}=1 \mid \mathscr{F}_{n}^{\alpha}\right\}$.

Assume that a Markov switching or jump process is independent of Brownian motion $W_{1}^{\mathrm{Q}}, W_{2}^{\mathrm{Q}}$. Combining both regime switching and jumps with the locally constant of the SV process $v$, with conditional density of $X_{n, \varepsilon}^{\dagger}$, from (62), we get

$$
\begin{aligned}
f\left(X_{n+1, \varepsilon}^{\dagger} \mid \mathscr{F}_{n+1}^{X^{\dagger}}, \alpha_{n}=k ; \Theta_{k}\right) \\
=\left(1-\lambda t_{n+1}\right) f\left(S_{n, \varepsilon}^{\dagger} \mid \mathscr{F}_{n}^{X^{\dagger}}, \alpha_{n}=j, \text { no jump; } \Theta_{k}\right) \\
\quad+\lambda t_{n+1} f\left(X_{n, \varepsilon}^{\dagger} \mid \mathscr{F}_{n-1}^{X^{\dagger}}, \alpha_{n}=i, \text { jump; } \Theta_{k}\right)
\end{aligned}
$$




$$
\begin{aligned}
= & \left(1-\lambda t_{n+1}\right) f_{\mathcal{N}}\left(x_{n}^{\dagger}, v_{n} ; b_{n, \varepsilon} \Delta_{n}+x_{n}, \widehat{v}_{n}^{2} \Delta_{n}\right) \\
& +\lambda t_{n+1} f_{\mathcal{N}}\left(x_{n}^{\dagger}, v_{n} ; b_{n, \varepsilon} \Delta_{n}+x_{n}+j \mu_{Y}, \widehat{v}_{n}^{2} \Delta_{n}+\sigma_{Y}^{2}\right) .
\end{aligned}
$$

Substituting (70) into (69) gives the conditional density of $X_{n, \varepsilon}^{\dagger}$ used in constructing the likelihood function for the data.

The set of the parameters $\Theta$ is estimated by the EM algorithm (for more details see [6]). The procedure for implementation to real historical data is as follows.

Algorithm 5. Consider the following.

(1) Estimate initial parameters of regime processes from historical data using a numerical scheme of model (58) with (48) to maximize the likelihood function (62) through a solver.

(2) Set an initial vector, $\Theta^{(0)}:=\left(r_{k}^{0}, \sigma_{k}^{0}, m_{k}^{0}, \beta_{k}^{0}, \mu_{Y, k}^{0}, \sigma_{Y, k}^{0}\right)$ for all $k \in \mathcal{S}$. Fix the maximum number of iterations $N$ and approximation level $\varepsilon>0$. Apply the EM algorithm to find filtered probability with density function of (70) and smoothed probability using data generated by SV $v_{n}$ of (48) as a variance process of discretization for approximation process $X_{n, \varepsilon}$.

(3) Generate the process $X_{n, \varepsilon}$.

\section{Application Example of Thailand SET Index}

In this section we provide numerical results from the proposed model. We consider a stock index for the Bangkok Stock Exchange of Thailand (SET) index from January 1, 2011, to February 2013. We fix $r$ and $q$ at 0.025 and 0.125 , respectively. The estimates for the proposed model (58) with SV (48) using daily data on continuously compounded SET index returns are $\sigma=0.7500, m=0.055, \beta=0.4500$ with $\mu_{Y}=0.2250$ and $\sigma_{Y}=0.1250$ and being fixed. The data cover the sample period, January 1, 2011, through February 15, 2012. In estimating the (58), we fix $\varepsilon$, somewhat arbitrarily, at 0.5 .

Implementation of the Matlab program following Algorithm 5. The log prices $X_{t, \varepsilon}^{\dagger}$ in the case of proposed model (58) are the base regime and the second regime representing the jump prices is given by i.i.d. log-normal distribution $\log \left(X_{t}, \alpha_{2}\right) \sim \mathcal{N}(0.3250,0.485)$. Calibration results for model (58) with two independent regimes fitted to the SET index log prices are displayed in Figure 2.

\section{Conflict of Interests}

The author declares that there is no conflict of interests regarding the publication of this paper.

\section{Acknowledgments}

This research is especially dedicated to the Department of Mathematics and Computer Science, Faculty of Science and Technology, Prince of Songkla University, Pattani Campus. And this research project has been financially supported by the Centre of Excellence in Mathematics, the Commission on

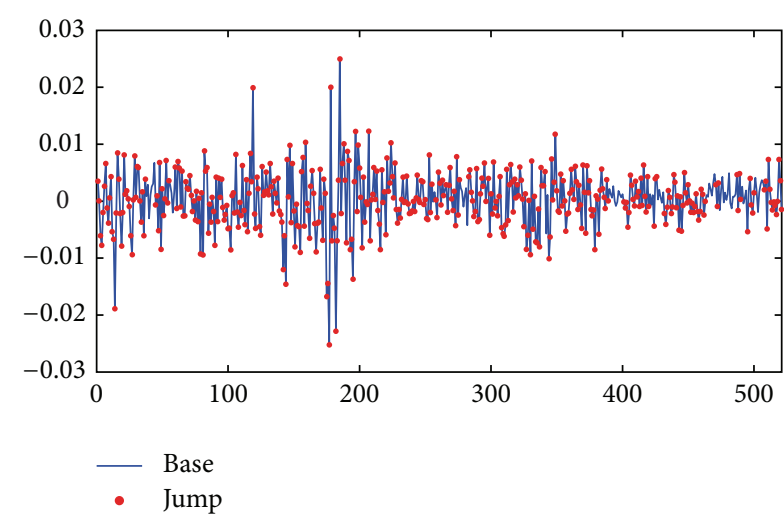

(a)

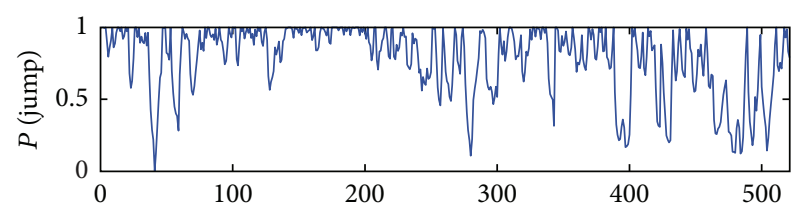

(b)

FIgURE 2: Calibration results for model (56) with two independent regimes fitted to the SET index log prices. The prices jump is denoted by dots in the upper panel and the lower panel shows the conditional probability of beginning of the jump regime.

Higher Education, Thailand, under the individual project at 2011, Grant no. RS-2-54-05-1.

\section{References}

[1] J. D. Hamilton, "A new approach to the economic analysis of nonstationary time series and the business cycle," Econometrica, vol. 57, no. 2, pp. 357-384, 1989.

[2] R. J. Elliott, L. Chan, and T. K. Siu, "Option pricing and Esscher transform under regime switching," Annals of Finance, vol. 1, no. 4, pp. 423-432, 2005.

[3] T. K. Siu, J. W. Lau, and H. Yang, "Pricing participating products under a generalized jump-diffusion model," Journal of Applied Mathematics and Stochastic Analysis, vol. 2008, Article ID 474623, 30 pages, 2008.

[4] M. Kalimipalli and R. Susmel, "Regime-switching stochastic volatility and short-term interest rates," Journal of Empirical Finance, vol. 11, no. 3, pp. 309-329, 2004.

[5] D. R. Smith, "Markov-switching and stochastic volatility diffusion models of short-term interest rates," Journal of Business \& Economic Statistics, vol. 20, no. 2, pp. 183-197, 2002.

[6] J. Janczura and R. Weron, "Efficient estimation of Markov regime-switching models: an application to electricity spot prices," AStA Advances in Statistical Analysis, vol. 96, no. 3, pp. 385-407, 2012.

[7] B. Øksendal and A. Sulem, Applied Stochastic Control of Jump Diffusions, Universitext, Springer, Berlin, Germany, 2005.

[8] N. H. Bingham and R. Kiesel, Risk-Neutral Valuation: Pricing and Hedging of Financial Derivatives, Springer Finance, Springer, London, UK, 2nd edition, 2004. 
[9] J. Duan, J. E. Gentle, and W. Hardle, Handbook of Computational Finance, Springer Handbooks of Computational Statistics, Springer, Heidelberg, Germany, 2012.

[10] B. F. Hanson, "Stochastic calculus of Heston's stochasticvolatility model," in Proceedings of the 19th International Symposium on Mathematical Theory of Networks and Systems (MTNS '10), pp. 2423-2429, Budapest, Hungary, July 2010. 


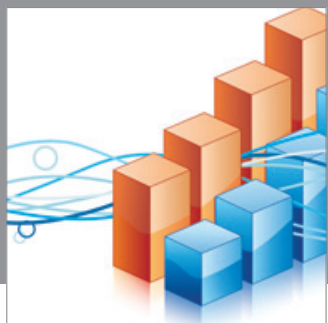

Advances in

Operations Research

mansans

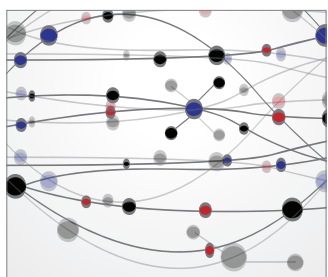

The Scientific World Journal
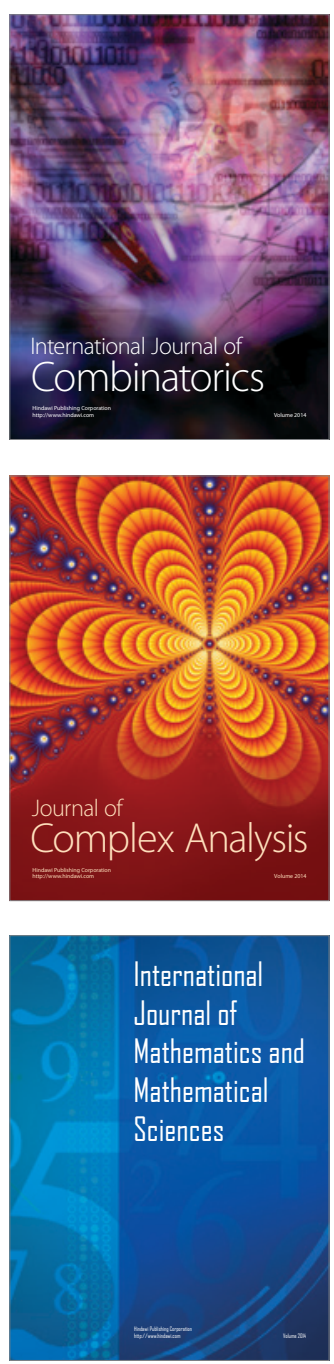
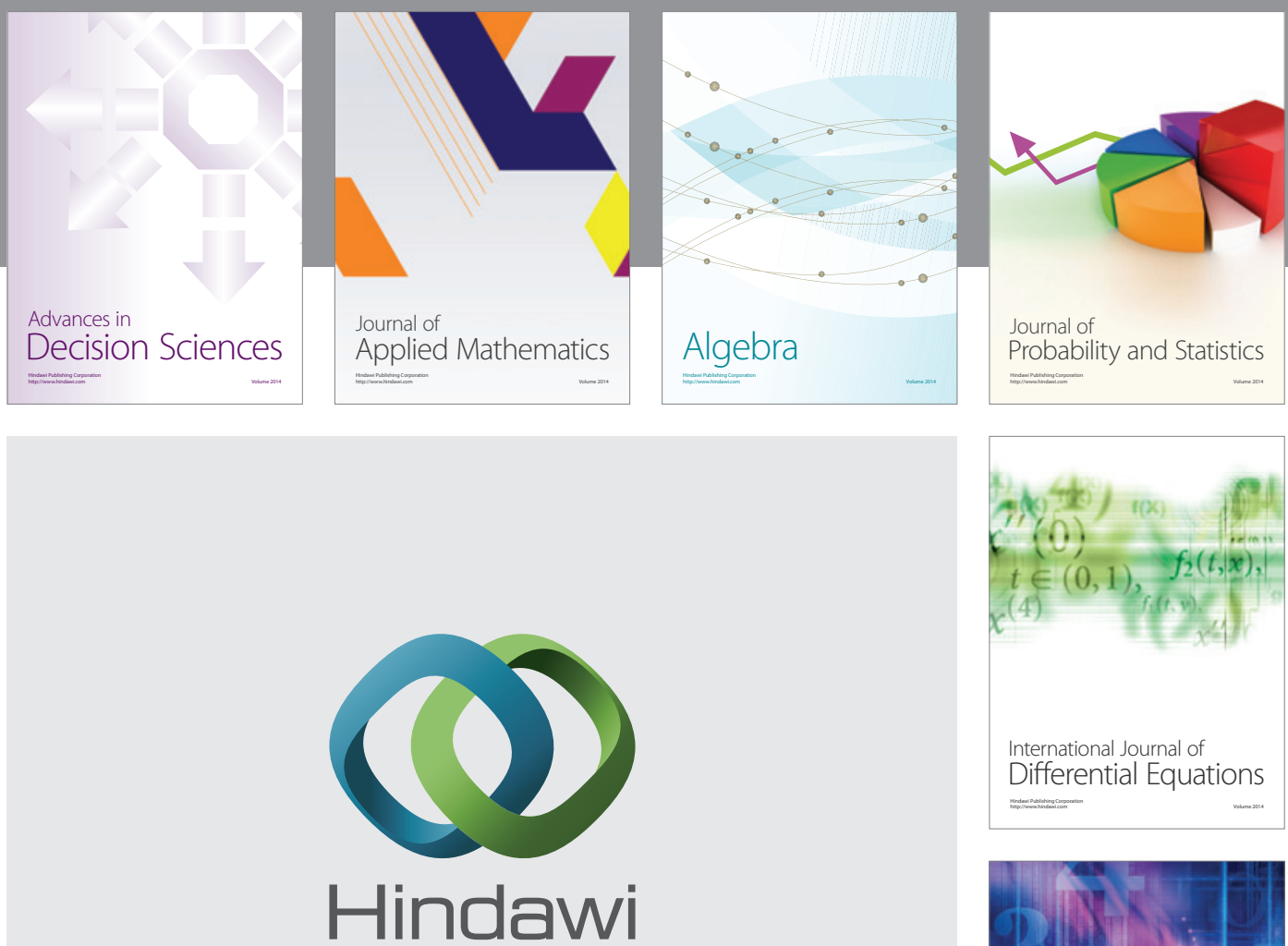

Submit your manuscripts at http://www.hindawi.com
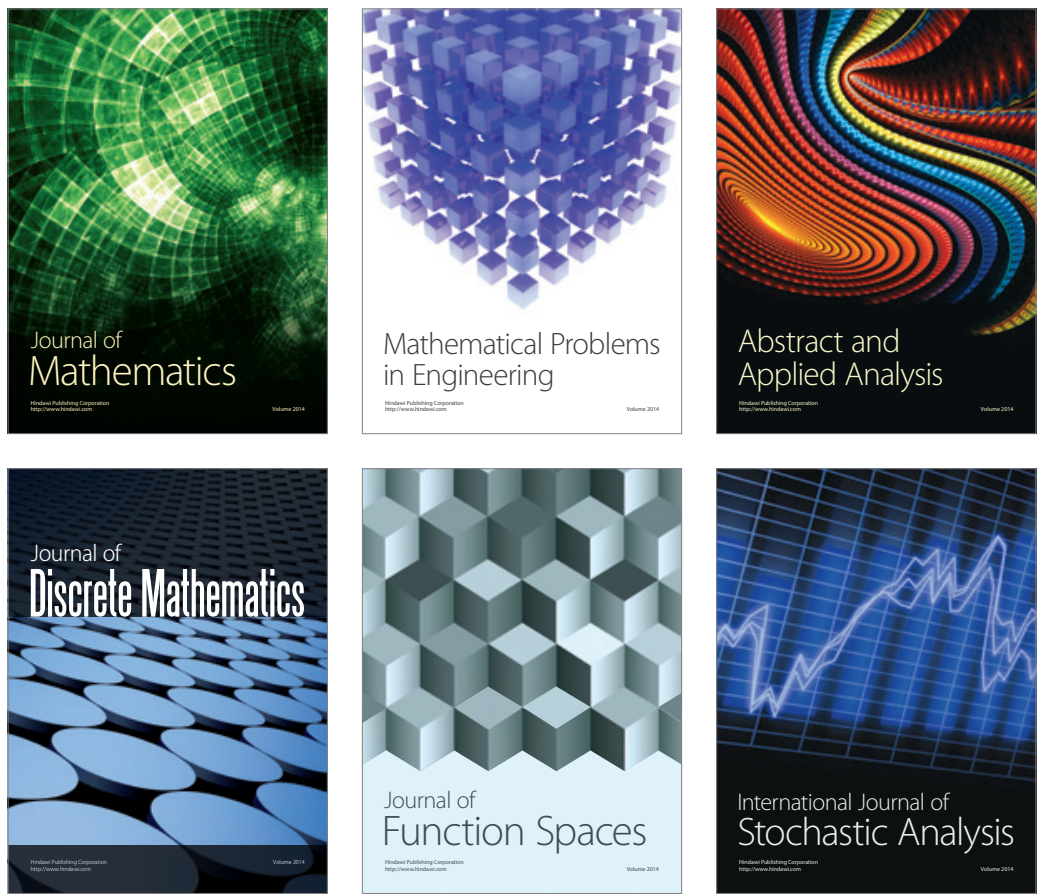

Journal of

Function Spaces

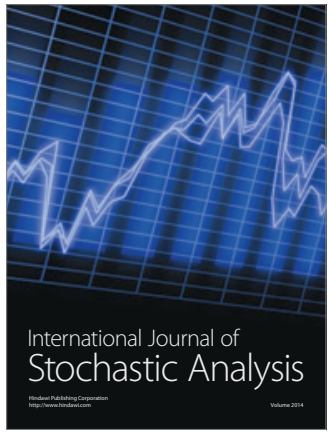

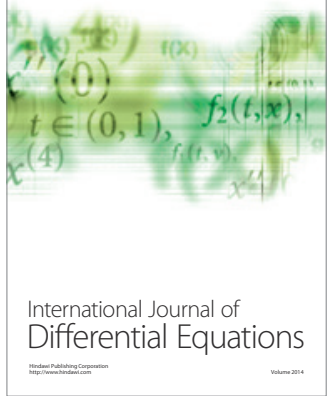
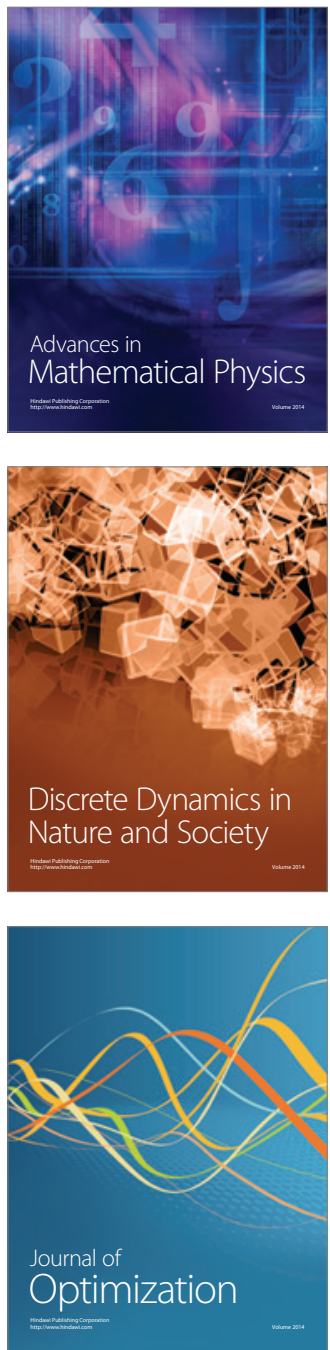